Vol. 9, Issue 4, April 2020

\title{
Medical Device Qualification Use
}

\author{
Sai Sruthi Gadde', Venkata Dinesh Reddy Kalli² \\ Ph.D. in Information Technology, University of Cumberlands, Louisville, Kentucky, USA ${ }^{1}$ \\ Research Scientist, Cardiac and Vascular Group, Medtronic, Tempe, USA ${ }^{2}$
}

\begin{abstract}
Before entering the market, the health authorities like the FDA must approve pharmaceutical products and medical devices. It is difficult to guarantee that the software is incorrect to prevent any effort to control medical information. All software is affected. But the correctness of medical experience is at least as critical in medical software as the correctness of the code itself. The medical software's contents can usually be measured, but the end-users have little time to do so or little incentive. The Internet allows non-professionals to provide commercial services. Several commercial platforms operate on the net for healthcare already. Since medical software is not guaranteed or regulated, anyone can sell medical software on the net. There is a risk that patients can use even though they are cautious enough not to use untested apps. In Finland, where more than $10 \%$ of the population uses the Internet on a weekly basis, there is a real problem. Inadequate services cannot be removed from the network and, therefore, high-quality services are necessary to direct users to use. The paper addresses various aspects of medical device evaluation.
\end{abstract}

Keywords: ICT,FDA, Medical software

\section{INTRODUCTION}

The rapid advances in ICT have allowed the development of new services for the public. Healthcare is one of the most critical regions. Medical experience is typically authentic worldwide, rendering the demand worldwide. Health systems and tools for decision-making are becoming essential instruments for medical professionals, but health information is still a concern of ordinary people. The health-telematics market is expected to be similar to that of the pharmaceutical industry today in 2010. People are curious to learn more about medicine in general and their illnesses in particular. The Internet now provides powerful search engines and a wide range of resources for medical knowledge, including free apps for everyone. In previous days, patients had restricted access to medical education as it was only available from the texts and specialist journals. Health information resources on the Internet are, however, most frequently accessed by individuals with medical experience, such as patients or family members. Anyone should have the right to place information on the Internet, but users should know whether or not qualified information is given. It is easier to say than to do. Health software is also available on the Internet. Technology, in particular, which provides patients with information on their treatments, may be harmful. The Internet provides tremendous promise in medicine in spite of these risks. Previously, only local medical applications were developed. There is now a way around the world of disseminating and selling healthy medical apps. When this is done cleverly, enough capital can be obtained to build reliable decision support systems that no one could conceive of only a decade ago. Medicine can be less dangerous than using computer software [1]. [2]. The state-of-the-art counseling cannot be offered because clinicians cannot consider relevant details. This is one of the key reasons for using healthcare decision-making support systems.

As the level of decision-making support improves, more medical decisions are based on machine suggestions. This will eventually lead to questions about the medical license. What is medical software going to play? Health professionals should be actively involved in the development of the knowledge society of a regulatory system for medical services. Measures that promote high-quality service output and reduce the risk of false information are required for the public. This paper explores the possibilities and benefits of medical device evaluation and registration.

\section{MEDICAL SOFTWARE}

Medical software is defined as any medical information software in this article. Knowledge can be interpreted as useful data. The number 37 is, however, info, but it becomes information when we say that the average body temperature is $37^{\circ} \mathrm{C}$. What about the MEDLINE and Science Citation Index databases?. Do medical skills involve them? Databases are sizeable indexed data sets. Contradictory data can exist for one subject. The database does not have the function of interpreting. The user is responsible for reading the data, looking at the data. Such databases do not turn information into expertise and do not previously meet my medical software requirements. Nevertheless, databases may be known as medical applications. Of example, the literature typically gathers databases of drug interactions, and findings about the nature of such drug interactions 


\author{
Vol. 9, Issue 4, April 2020
}

are based on an expert summarizing all the knowledge that is available in relation to the topic. The enormous number of new data pubs generated annually in biomedical science will contribute to the use of medical education. This is a meta-analysis that increases the value of users' data. Details are lost in the processing of more information, but the information is acquired. It can not be achieved automatically since a great deal of domain expertise is needed to conduct the job.

Typical tests for medical applications are decision support systems. However, there are many applications that are not typically re-established as decision support systems but include medical software features. Frameworks for the writing of electronic prescriptions, risk assessment frameworks for some diseases, and applications with the creative presence of clinical knowledge (e.g., multi-dimensional clustering techniques) are just some examples.

Throughout recent years there has been a more positive attitude amongst healthcare professionals towards the use of computers throughout healthcare. The development and preparation of computer systems requires more professional practitioners. The technology itself is now known as not a concern. Computers in healthcare can be used to provide high-quality, affordable care [2-5]. Therefore, scientifically, the steps to guarantee the quality of medical applications have become more critical. [6,7].

\title{
III. CHANGING ROLE OF MEDICAL SOFTWARE
}

At the moment, the authorities monitor only device-related applications. Nevertheless, it is challenging to describe device-related applications. The latest trend is that laboratory machines have links to the network to interact with servers. In the near future, those devices will be intelligent on a server that serves all devices worldwide. For a computer maker, this is a very inexpensive approach. The Company can detect an error more quickly than ever when there is a software error. Computer updates are affordable and straightforward because only one or many machines have to update the device. Since the knowledge is not built into the products, a new industry will provide smart software for analyzing the data collected from products. Imagine one additional move. Let us presume that independent software developers are developing applications for clinical laboratories that read data from multiple devices and hospital information systems. These applications cannot be classified as applications connected to computers. She is her own commodity. To sum up, software relating to computers will become obsolete, and no distinction between software relating to faults and other software can be made in the future.

The support systems for clinical decision-making would be more nuanced as they can gather data from different sources. There is also a decision support module connected with the analyzer that is held in device-related applications in some laboratory systems for thyroid diagnostics research. More specialized devices that extract medicine from an electronic medical record and laboratory test results from the analyzer are used in some laboratories. This enables superior decision support in thyroid diagnostics to be developed. At least as important as the older program is this latter. Yet because device-related software is not involved, there is no official evaluation of the program. A comprehensive quality control for many decision support software is required to leverage the full power of electronic patient records in decision support applications.

\section{EVALUATION OF MEDICAL PRODUCTS}

a. $\quad$ The need for thorough evaluation: an example

A year ago, users found a minor mistake in the program for biological testing for the syndrome of Down[8]. The program gave Down's syndrome too little odds that could have contributed to too low detection rates. This was an obvious mistake, but luckily the amount of premature Down's syndrome births didn't have a major impact. The error was nevertheless recorded in The Lancet, and it was quite significant. It demonstrated how critical the medical information used in the program is to be thoroughly evaluated. The diagnosis of thousands of patients a day can be taken with apps. A flaw in a system, as the number of patients seen by one physician, may cause much more harm than a single physician's comprehension.

There are actually two primary groups of medical products:

\section{Medical devices Drugs}

Medical software is evaluated similarly to medical devices, particularly for historical reasons. The assessment is also highly technical, and there has to be no detailed evaluation of the medical expertise in the applications. In addition, most scientific papers are often better evaluated for medical contents than the device-related software! The U.S. is ahead of Europe in the implementation of health care technologies. The FDA started regulating device-related software from 1976[9-12], even most experienced in the regulation of medical software come from the USA. Two types of medical device software were primarily developed: software for the development of medical devices and software for medical devices. Software in the sense of medical devices, in particular, prompted a vigorous debate of legal status and ethical issues as machines replaced the opinion of health care 
professionals [13].

Changes to the Food and Drug Act for the 1990 Medical Device culminated in a significant shift in medical device control. The 1990 Act replaced the prior emphasis on pre-market authorization with a focus on postmarket control. The report of FDA product defects which caused injury or death in hospitals and other institutional users. They also had to disclose the manufacturer's product defects. The new legislation specifically has an impact on software Regulation as the question of software quality could far more easily be tackled than the legislation of 1976[14]. The Act stipulated that system approval would immediately be revoked, faulty goods recalled, and civil penalties levied for violators. Such factors have combined lead to increased FDA oversight of medical product acquisition and use and, in particular, to a greater interest in finding uncontrolled producers.

Ten years ago, there were significant gaps in medical tech policies in America and Europe. The American and E.U. European approaches Professor Beier compared[15]. The FDA regulation is very stringent, and faulty goods manufacturers run high prosecution risk. The FDA prevented the scientists from designing medical applications. Many software is, in fact, deliberately classified as 'non-clinical or search-only' only to escape legal liability [16]. The FDA's medical program policy[17-21] has been strongly negative in much of its studies, and its approach has been defended by the FDA [22].

The 1993 directive[23] basically has the same policy in Europe as the FDA Act for device-related applications. The assessment starts in this Guideline with a risk classification in three key classes of devices and associated applications. The assessment is primarily theoretical and relies less on the medical expertise itself. I consider medical expertise as the intelligent part of the software in this document that interprets measurements and test results.

The FDA Medical Technology Policy created a new wave of frustration as medicine became useful in neural networks[24-26]. Previously, the FDA rules mandated that consumers understand the medical information found in the computer applications. That is not the case for neural networks, and recent changes have been made to the FDA guidelines for medical device-related applications. In 1997 it came into practice.

\section{EVALUATION OF HUMAN DRUGS AS A MODEL FOR MEDICAL SOFTWARE}

It cannot be as precise as in scientific equipment in a biological environment. Statistical measurements are based on facts of biological systems. A drug is first tested on animals, then healthy volunteers, and then a patient population during its growth. Before a drug is approved into clinical use, the results must be demonstrated with a certain degree of trust. Medicines typically re-registered, which are found ineffective.

The pharmaceutical industry could learn a lot from the medical knowledge community. Clinical studies will be carried out to demonstrate that the use of medical software in health care is useful. There are some important differences that make software clinical trials much tougher than medicines. The immediate effects of drugs on patients are observable. The effect of medical software on clinical decisions will result in tangible patient results. The precise outcome of the use of software can be difficult to determine. Better standards of treatment and/or lower costs may result, but these metrics are highly contentious. The clinician's unit of research is the practitioner rather than the patient as decision support software. This makes it difficult to hire enough people to make statistically based claims. The time of the specialist is much longer than the computer's CPU time. Consequently, there are situations where computerized option support contributes to faster decision-making, where costs can be significantly reduced. The reality that only a slight decrease in the quality of health care because of the substitution of clinicians by machines in some cases appears to be a matter for clinicians and science experts.

This may, however, change if the cost of healthcare for society is insupportable. For example, where computerized decision-making support can cut costs, are the substitution of mammographic radiologists by software, replacement of clinicians in anticoagulant care for the warfarin dose, and replacement of clinicians in the analysis of 24-hour ECG records.

Technology can typically provide decision support in the final decisions the physician makes. Technology for ECG detection is a common example of this. The doctor uses the ECG as well as its explanation. Computer-aided QRS complex analysis helps clinicians to track the 24-hour recording much quicker in the 24-hour recording process.

In future research, it is always difficult to compare various medical treatments or diagnostic procedures. Because the illness examined is unusual, statistical variations between therapies or diagnostic procedures cannot be obtained. The question is even more significant with medical devices. The program must be modified to prevent outdating if new release information is written. Technology can also be upgraded many times a year. It could ruin future research but must be acknowledged. The condition is probably worse. When you implement new information in an assessed system, you will prove that the software is as qualitative as before. Assuming that is the case is the skepticism of new technologies for software development.

Is there any point in testing the software if it's challenging to evaluate medical software and if it changes 


\author{
Vol. 9, Issue 4, April 2020
}

continuously? Surely yes. Yes. When medical assessment software is not usable, it may be sold as black boxes and used as crystal balls. There are several crucial clinical thumb laws in medicine. Creating decision support software on the basis of these rules is very simple. It may take years for a rare disease to see that the product has no intelligence and that software programmed intelligence does not necessitate a machine. The methods of choice for a variety of complex pattern recognition tasks have proved to be modern non-linear techniques as neural networks and fuzzy logo. The knowledge is portrayed in these advanced methods in a manner which the user can not understand. It is also much easier to cheat with neural networks than with systems based on law. The user finds it very difficult to determine whether or not the software is useful.

This is also important to carry out post-marketing surveillance in the field of medical applications for 'pharmacovigilance,' i.e., tracking and reporting potential adverse medical reactions between the patients to the health authorities. In regard to software 'function vigilance,' the authorities are told about the findings any time a software error is reported. You may then request corrections, or in severe cases, if the numbers of problems experienced are high, the provider can call back the program. This in itself is difficult, because the software can easily be copied and "illegal" copies may still be used, particularly when the users not censored by the provider are not identified.

\title{
VI. WHO NEEDS EVALUATION?
}

\section{a. Users}

Medical software assessment shall be useful to those in the field. As the healthcare industry replaces paper-based documentation with electronic medical records, the demand for good health information will grow rapidly. Then, when combined with supporters, there would be little extra effort needed by physicians in using applications other than the EPR. The flash end for users is to find good applications. When manufacturers want to require applications for good product certificates to be reviewed by health authorities, it will be much easier to scan items, and a WEB server would be available to help consumers locate the applications they want searching for. The authority should also document all the problems that occur when using any program (vigilance history) and notify users of the test items.

\section{b. $\quad$ Software developers}

The certification will also support app developers. There is still a deep divide between medical device research publications and their use in clinical practice. It is difficult for developers to locate other interested users. Consumers don't know the latest applications, on the other hand. But when users find software that meets their requirements, they can have trouble depending on the program. Assessing and authorizing an application is likely to create a critical mass of users to make the program profitable. Over time the output would increase as abundant sources for further growth were allocated.

\section{c. $\quad$ Device manufacturers}

As previously stated, the production of decision support software on their devices is always challenging for device manufacturers. This is not because of the absence of good advocates, but rather because of the lack of real patient material. In the provision of medical information and patient essay data for medical applications, large hospitals should play a key role. It could be a wise option for a computer maker to concentrate on their core business, build equipment, and buy the software from another firm.

\section{d. $\quad$ Insurance companies}

Health care benefits are also being pursued by insurance providers and regional health agencies. The prices of new medicines and therapies in developed countries are growing more rapidly than the GNP. The issue has been overcome with the use of information technology. In this sense, telemedicine was one of the keywords. There is little evidence. However, that telemedicine will reduce healthcare costs. Human intelligence and time are still expensive. The possibility is also present, which is not cost-effective for the general healthcare system, that specialists are more consulted with telemedicine patients and G.P.s. The situation is somewhat different for medical applications. If some portion of the work of the physician can be substituted for health services, costs are more likely to be minimized. Health insurance would possibly in the future cover at least part of the expense of using medical software, which has proven that it reduces healthcare costs. An independent assessment and registration authority will assist insurers in determining whether the program can be supplied or not. A simple connection to drugs once again exists. The European Agency may recognize human medicines on the market, but the national health policymakers will be responsible for determining if they pay part of the costs of medicinal medicine or all of it. For medical applications, the same layout is suitable. Careful evaluation of the program is a costly process. Unless every nation had its own assessment procedure, it would be a waste of resources. This does not mean that, due to the variations in epidemiology and medical technology, the findings of a study in one 


\author{
Vol. 9, Issue 4, April 2020
}

country are easily transferable to another country. Finally, all the available information must take account of the ultimate decision to promote the use of a software product at a national level.

$e$.

Patients

The Internet has transformed the tech markets. Every school child is now able to use the Internet to make medical information accessible. Health software is an internet sales company. In the very near future, the Internet trade will rapidly expand, and the services provided over the net will be charged using tools. Technology for patient treatment on the Web is now widely available. The writers note that the program is not intended for clinical use in order to escape legal liability. How often, patients and doctors prefer to use patient care apps. A good example is a simulator which estimates insulin-dependent patients with diabetes. Clinicians not allowed in the course of their operations to use the program told patients of the possibility of accessing their program to assist with insulin treatment from an internet address. This strategy is far more dangerous than a software clinician. The patients have no prior information required for determining whether or not the software's advice is accurate. The results can, sadly, be fatal. It will also be hard to tell whether the program makes a mistake if the patient denies saying to his doctor that he is using any program.

Another example is a demonstration of an emergency service dispatch system built for dispatchers accessible on the Internet. It is advised that a cardiac ambulance be sent or not to a thoracic emergency. The danger is apparent both in the case of false negatives where patients may die because of the emergency service's lack or too late arrival and in the event of false positives that can impair treatment when necessary and the rise in healthcare costs.

To purchase a medical textbook and program those health applications without the requisite medical expertise, it would be incredibly simple for a computer programmer. Attempts to remove incorrect content on Inter-net were not very effective, and the distribution of medical information on the Internet can not be restricted. However, we have to understand that the Internet provides doctors and patients with strong medical information. We will help users assess the current services and provide information on high-quality services and warn to potential risks in some software applications. This does not completely solve the issue, but it is an essential step to improve the safety of patients. Auto security, such as 'Safety On the Net' is a first move [27].

\title{
VII. REGISTRATION OF MEDICAL SOFTWARE
}

The FDA's effect on the medical tech industry is too powerful in the U.S., and it is worried. There is a danger that the assessment of medical applications would result in strict FDA control. This means that if the product is not considered useful by the FDA, it will be prohibited for commercialization in the worst-case scenario. This approach has proven to be important in medications and medical devices because the patient might not be in a position to determine the risks alone. For medical software, it is difficult: in medical practice, objective truths are rare; many technologies are so unique that only a limited number of experts recognize their usefulness.

But a definitive decision can not be made: yes or no. A formal report on different aspects of the program will be much more useful. There is definitely a long list of problems to consider, and not all of them fit all the applications. I think that all publicly available medical applications, commercially or otherwise, should contain specific user details, including the above aspects.

\section{CONCLUSIONS}

Over the next few years, funding for the selling of medical decisions on the Internet will burst. Such facilities would not only be used by medical professionals but also by laypeople. Any applications on the Internet cannot be censored. Nonetheless, an independent body should make it compulsory to register the service. For many factors, this will be helpful. Second, after marketing software, surveillance is possible as users may contact the authority with software errors or other complaints. Within the next few years, funding for professional decision taking will burst on the Internet. Such facilities should not only be used by medical practitioners, but also by the laity. Any applications on the Internet can not be censored. Nevertheless, an independent body must make it mandatory to register the service. For many factors, it would be helpful. First, after marketing, software monitoring is possible because users may contact the authority with software errors or other grievances.

\section{REFERENCES}

[1]. A.W. Hafner, A.B. Filipowicz, W.P. Whitely, Computers in medicine: liability issues for physi- cians, Int. J. Clin. Monit. Comput. 6 (1989) $185-194$.

[2]. H.M. Schoolman, Obligations of the expert system builder: meeting the needs of the user, M.D. Com- put. 8 (1991) 316 - 321.

[3]. P. Hammond, A.L. Harris, S.K. Das, J.C. Wyatt, Safety and decision support in oncology, Methods Inf. Med. 33 (1994) $371-381$.

[4]. J.C. Wyatt, Clinical data systems, Part 3: Develop- ment and evaluation, Lancet 344 (1994) 1682 - 1688 


\title{
International Journal of Advanced Research in Computer and Communication Engineering
}

\author{
Vol. 9, Issue 4, April 2020
}

[5]. J.C. Wyatt, Hospital information management: the need for clinical leadership, Br. Med. J. 311 (15) (1995) 175 -178.

[6]. P.S. Cosgriff, Quality assurance of medical soft- ware, J. Med. Eng. Technol. 18 (1994) 1 - 10.

[7]. R. Shaw, Safety-critical software and current stan- dards initiatives, Comput. Methods Progr. Biomed. 44 (1994) 5 - 22.

[8]. P. Cavalli, False negative results in Down's syn- drome screening, Lancet 347 (1996) 965 - 966.

[9]. Food and Drug Administration (FDA): The May 28, 1976, Medical Device Amendments to the Fed- eral Food, Drug and Cosmetic Act.

[10]. C. Holden, Regulating software for medical devices [news]., Science 234 (1986) 4772.

[11]. V. Brannigan, The regulation of medical computer software as a 'device' under the Food, Drug, and Cosmetic Act, Comput. Methods Progr. Biomed. 25 (1987) $219-228$.

[12]. R.R. Murfitt, United States government regulation of medical device software: a review, J. Med. Eng. Technol. 14 (1990) 111 - 113.

[13]. R.A. Miller, K.F. Schaffner, A. Meisel, Ethical and legal issues related to the use of computer programs in clinical medicine, Ann. Intern. Med. 102 (1985) 529-537.

[14]. V.M. Brannigan. Software quality regulation un- der the Safe Medical Devices Act of 1991: hospi- tals are now the canaries in the software mine. Proc. Annu. Symp. Comput. Appl. Med. Care 1991, $238-242$.

[15]. B. Beier, liability and responsibility for clinical software in the Federal Republic of Germany, Comput. Methods Progr. Biomed. 25 (1987) $237-242$.

[16]. L.J. Brindza, FDA regulation of computerized cy- tology devices, Anal. Quant. Cytol. Histol. 13 (1991) $3-6$.

[17]. C.J. McDonald, Medical software regulation: why now? [editorial]., M.D. Comput. 3 (1986) 7 - 8.

[18]. M.L. Norton, E.V. Norton, Medical computers from the manufacturer's viewpoint, Comput. Methods Progr. Biomed. 25 (1987) 229 236.

[19]. K. Harby, New FDA software proposals: effect on nuclear medicine? [news]., J. Nucl. Med. 29 (1988) $588-589$.

[20]. J.I. Schwartz, J.L. Fink, Legal issues associated with pharmacokinetic software, Am. J. Hosp. Pharm. 46 (1989) 120 - 124.

[21]. G.E. Gamerman. FDA regulation of biomedical software. Proc. Annu. Symp. Comput. Appl. Med. Care $1992,745-749$.

[22]. C.S. Furfine, The FDA's policy on the regulation of computerized medical devices [comment]., M.D. Comput. 9 (1992) 97 - 100.

[23]. Council of the European Union. Directive EC/42/ 93 on device related software.

[24]. J.F. Place, A. Truchaud, K. Ozawa, H. Pardue, P. Schnipelsky, Use of artificial intelligence in analytical systems for the clinical laboratory, Clin. Biochem. 28 (1995) $373-389$.

[25]. J.J. Forsström, K.J. Dalton, Artificial neural networks for decision support in clinical medicine: areview, Ann. Med. 27 (1995) 509 517.

[26]. J. Järvi, J.J. Forsström, A. Koski. The NE- SUMED Project-Case Studies of Computational Intelligence in Medicine. TOOLMET 96; Oulu, Finland, April 1 - 2, 1996.

[27]. Kalli, V. D. R. (2020). Technology Engineering for Medical Devices - A Lean Manufacturing Plant Viewpoint. International Journal of Advanced Research in Computer and Communication https://doi.org/10.17148/IJARCCE.2020.9401http://www.hon.ch 